\title{
Cracked Tooth Syndrome - An Elusive Diagnosis
}

\author{
Dr.Shalini $\mathrm{H}^{1}$, Dr. Manish ${ }^{2}$ \\ (Department of endodontics, Saveetha Dental College, India) \\ (Department of endodontics, Saveetha Dental College, India)
}

\begin{abstract}
Cracked tooth syndrome is a major diagnostic challenge in clinical practice. It presents with bizarre of signs and symptoms which is frustrating for both the dentist as well as the patient. Successful diagnosis lies in the awareness of its presence and appropriate diagnostic tests. This article will explain the various diagnostic procedures to detect cracks, as well as to diagnose its extent through the nature of pain elicited, followed by appropriate treatment modalities.
\end{abstract}

Keywords: Bite tests, crack detection, cusp fracture, pain mechanism, vertical root fracture,

\section{Introduction}

The term cracked tooth syndrome was first introduced by Cameron in $1964^{[1]}$. It refers to an incomplete fracture of a vital posterior tooth that involves the dentin and occasionally extends into the pulp ${ }^{[1]}$.Cracked tooth was termed as a syndrome since it is often associated with a wide range of symptoms includingsensitivity to cold, pain on chewing and on release of pressure, symptoms of pulpitis when pulp is involved, periodontal disease if fracture extends to the root ${ }^{[2]}$.

The detection and diagnosis of cracked tooth is a major challenge in clinical practice ${ }^{[3]}$. A wide knowledge about its presence causes and range of clinical presentation plays a significant role in its diagnosis ${ }^{[4]}$. Diagnosis doesn't mean clinical visualisationalone, as crack detection alone cannot assess the status of the pulp or Periapical tissues ${ }^{[5]}$. Cracks are findings only; they are not to be considered as a pulpal or Periapicaldiagnosis ${ }^{[5]}$. Hence, this article will show the methods of crack detection and its diagnosis followed by appropriate treatment modalities.

The Basic steps in crack detection includes,

\section{Diagnosis}

\section{Dental history}

Patient's history should be assessed very carefully. History of any masticatory accidents, Para functional habits like bruxism, past dental treatment(iatrogenic factors) ${ }^{[6]}$, dietary habits, beetle nut chewing, trauma or accidents may give a clue to our diagnosis. History of previous cracked teethdue to many anatomical ${ }^{[7]}$ and developmental factors ${ }^{[6]}$ may predispose teeth to crack. History ofperiodontal disease with extensive bone loss in the site can cause increased stresses on dentin predisposing the tooth to rack $^{[8]}$. Cracks in root canal treated teeth are often due to size, design and placement of posts ${ }^{[5]}$.

\section{Visual examination}

Extra oralexamination, shows enlarged masseter muscles due excessive stress on mastication or Para functional habits like bruxism ${ }^{[9]}$. Intraoral examination should be done using rubber dam in a clear and dry field. Visualisation of cracks becomes easier in Contrasting and brighter colour of rubber dam ${ }^{[10]}$. Check for the wear facets.Brown studied that teeth subjected to thermal cycling between 90 to 140 degrees had severe cracking ${ }^{[11]}$. . $h e c k$ for abfraction lesions. Abfraction lesions are found to be associated with $94.5 \%$ of occlusal wear facets. Excessive occlusal loading has been suggested to be a possible cause of fracture in some unrestored teeth with abfraction lesions ${ }^{[1]}$. Steep cusps andwedging effect of cusp fossae relationship create hammer and anvil "chewing effect" that can propagate cracks ${ }^{[13]}$. Wide and deep restorations ${ }^{[2]}$ also predispose to cracks. Magnifying loupeswith illumination enhances our vision in crack detection ${ }^{[7]}$.

\section{Tactile examination}

Palpate the gingiva around the tooth for any dehiscence or fenestration typical of vertical root fracture. Palpation may show tenderness and swelling over the root and in Periapical region in cases of vertical root fracture.Running the tip of a sharp probe along the tooth surface produces a clicking sound when it passes over the fracture line $e^{[14]}$. 


\section{Bite tests}

Rubber wheel, wooden stick or tooth sloth fracture detector can be placed on the cusp of the suspected tooth and ask the patient to bite down with moderate pressure and then release. Pain during biting or chewing is a classic symptom $^{[2,15]}$.

\section{Periodontal probing}

Localised bone loss can result in excessive stresses on the exposed dentin predisposing crack formation ${ }^{[8]}$. Careful periodontal probing in small increments will restrict the movement of the probe thus helps to locate a crack $^{[5]}$. A deep narrow isolated periodontal pocket is present in cases of vertical root fracture ${ }^{[3]}$.

\section{Staining}

$2 \%$ Methylene blue , $0.25 \%$ sodium fluorescein ophthalmic solution,snoop caries detecting dye can be used to disclose a crack on surgically exposed roots or crack in the cavity after removal of the restoration. Wright, in his study proved that methylene blue combined with Transilluminaton was the best in crack detection ${ }^{[16]}$.

\section{Transilluminaton}

A fibre optic light source combined with magnification helps in visualisation of cracks on tooth surface ${ }^{[16]}$. The light beam is directed in a horizontal direction perpendicular to the plane of the suspected crack. Cracks will block the light beam from reaching the part of the tooth beyond the fracture, whereas sound teeth will transmit light through the crown ${ }^{[3]}$.

\section{Radiographs}

Radiographs are not conclusive in detecting cracks ${ }^{[17]}$ inthe earlier stages unlessthere is separation of segments of the tooth. Typical radiographic features for vertical root fracture includes

8.1 When a well condensed root canal filling appears very close to one wall of the root ${ }^{[19]}$.

8.2 Vertical radiolucent line running across fracture line ${ }^{[19]}$.

8.3 Extrusion of root canal filling material through the apex due to vertical root fracture may show a tangle of accessory points at the apex ( apical spaghetti) ${ }^{[19]}$

8.4Wide enlargement of periodontal ligament space through the whole length of the $\operatorname{root}^{[18]}$.

8.5 Pitts and Natkin described halo like radiolucency running around the tooth ${ }^{[20]}$.

8.6 Step like bone defect may be seen when fracture runs obliquely across the root ${ }^{[20]}$.

8.7 V shaped radiolucency which is widest at the crestal bone and narrowest towards the $\operatorname{apex}^{[20]}$.

8.8 Thickened periodontal ligament space or a diffuse radiolucency with J-shaped appearance in the apical region $^{[19]}$.

Ultrasound imaging system is capable of imaging cracks in simulated human tooth and would be a possible diagnostic tool in the future ${ }^{[21]}$.

\section{Restoration removal}

Bulky restorations or restorations with pin head designs ${ }^{[22]}$ should be cautiously examined for a crack. Removal of restorations allows for visual examination of the crack. Marginal ridges should be closely examined for a $\mathrm{crack}^{[3,15]}$. Thin marginal ridges predispose to cracks. Magnifying loupes and staining the tooth can aid in crack detection.

\section{$10 \quad$ Surgical assessment}

Surgical exploration remains the last option when all the other diagnostic means had failed to detect a crack. This consists of elevation of a full thickness flap and examination of bone and roots directly under high magnification and illumination ${ }^{[3]}$. But, the patient has to be fully informed that this is a diagnostic procedure only. Performing diagnostic surgery can visualise cracks along the root surfaces thus helping in early detection of untreatable situations avoiding the need for any complex treatment in hopeless cases.

\section{Mechanism of pain}

Detection of crack is inconclusive in the diagnosis and treatment. Visualisation of a crack in the tooth doesn't show its extent. Understanding the nature of pain ,will aid in the assessment of the extent and direction of the crack. Luebke ${ }^{[23]}$ suggested the following terms to diagnose pain from a cracked tooth.

1. Dentinal pain- sharp pain of short duration.

2. Pulpal pain - The spontaneous, deep, radiating pain produced by thermal shock to an inflamed pulp.

3. Periodontal pain- The aggravating and throbbing pain. 
Incomplete cuspal fracture results in dentinal pain due to rapid movement of dentinal fluid in the dentinal tubules according to the "Hydrodynamic theory of dentin sensitivity" proposed and investigated by Brannstrom ${ }^{[24]}$. Occlusal forces can cause cuspal flexure which produces thermal changes, air, evaporation, osmotic stimulation and increase in hydrostatic pressure resulting in rapid movement of dentinal fluid ${ }^{[25]}$. As a result, the A delta fibres in the vicinity of the odontoblastic processes and pulp-dentin border gets stimulated producing sharp pain of short duration indicating of a vital tooth.

Brief sharp pain of short duration but of lower threshold may be due to slight pulpal inflammation due to the infiltration of bacterial toxins into the pulp through the crack. This may be due to the stimulation of Adelta fibres whose stimulation threshold is lowered during inflammation.

Stimulation of $\mathrm{C}$ fibres due to inflammation, heat and mechanical deformation produces pain which is dull, aching and continuous throbbing nature or arise spontaneously and last for minutes or hours ${ }^{[25]}$. These fibres are activated due to infiltration of bacterial toxins into the pulp or fracture extending to the pulp or prolonged application of heat.

Incase of painful pulpitis, there is activation of both A-delta fibres and $\mathrm{C}$ fibres resulting in severe sharp painfollowed by prolonged dull ache radiating throughout the jaw ${ }^{[25]}$.

\section{Classification and treatment plan}

Followed by the diagnosis, a proper classification is essential for appropriate treatment plan. According to which longitudinal tooth fractures are classified according to the AAE (American association of endodontics ${ }^{[5]}$

1.

2. Class B - fractured cusp.

3. Class $\mathbf{C}$ - cracked tooth.

4. Class D- split tooth

5. Class E- vertical root fracture

1. Class A- Craze lines are present in most adult teeth. They are mainly present crossing marginal ridges extending along the buccal and lingual surfaces of posterior teeth. They affect only the enamel. Craze lines in anterior teeth are long and vertical.

Treatment- They are asymptomatic except for aesthetic concerns. Hence no treatment is needed.

2. Class B-Cusp fractures has the best prognosis of all the cracks, especially when the crack limits above the gingival attachment. This occurs mainly due to lack of cusp supportdue to weakened marginal ridge as a result of Restoration or caries. Usually only one cusp is affected(peripherally located crack). Patient presents with mild sensitivity to temperature changes and bite tests will elicit brief sharp pain especially on the release of pressure (hydrodynamic theory). The pulp remains vital.

\section{Treatment $^{[26]}$}

Restoration is removed. Mostly the affected cusp breaks off with relief of symptoms. Vitality of the tooth is checked (reversible pulpitis). Temporary restoration with a stainless steel band is given to the affected tooth. When symptoms resolve, the tooth can be restored with a full crown that covers the crack margins .If symptoms persist (irreversible pulpitis), Root canal treatment is necessary followed by full crown.

\section{Class C}

Cracked tooth are more centred than cusp fracture(centrally located cracks), therefore are more likely to cause pulpal or periradicular pathosis when it extends apically. Patient gives a history of acute pain on chewing or sharp brief pain to cold. He may have thermal sensitivity that persists after removal of stimulus or slight to very severe spontaneous pain consistent with irreversible pulpitis orpulp necrosis. Restoration removal or staining the tooth helps in visualisation of the crack. Gently test for the movements of the segments to differentiate it from fractured cusp. A fractured cusp or split tooth may break off under slight pressure while the segments of cracked tooth will remain in place.

\section{Treatment planning ${ }^{[25]}$}

Check for the vitality of the tooth. In case of reversible pulpitis continue as class B. If it is irreversible pulpitis, temporary restoration is given with stainless steel band. Endodontic treatment is indicated followed by a full crown to bind the cracked segments and protect the cusps. However before proceeding with treatment, other factors should also been taken into consideration 
1. Periodontal status need to be evaluated by periodontal probing and radiographic examination before commencing with further treatment. Deep probing indicates adverse prognosis. Vertical or furcation defects indicates severe crack.

2. Endodontic access is done to determine the extent of the crack in the pulpal floor. If the crack runs across the full width of the floor, the prognosis is poor. In this case extraction may be considered.If the crack line extends buccolingually in lower molar or involving single root in upper molar, root resection can be done. Staining, Magnification and illumination will confirm the presence of a crack on the pulpal floor. If the crack is partially visible on the pulpal floor, tooth may be bonded with orthodontic band to protect it from further damage till the completion of root canal therapy. If symptoms resolve the tooth is permanently restored or else tooth is extracted.

\section{Class D}

Split tooth is the final stage of crack progression. Prognosis is very poor to hopeless. Crack extends mesiodistally involving both the marginal ridges and two segments can be separated using a probe ${ }^{[14]}$. Patient complains of pain on chewing and soreness of gums on the affected area.

Treatment plan ${ }^{[26]}$ : Extraction of the tooth is indicated.

\section{Class E}

Vertical root fracture (VRF) mainly occurs on root canal treated toothdue to overzealous instrumentation, post placement, condensation during obturation, poorly designed dowels ${ }^{[3]}$. It begins in the apical third of the root usually in the buccolingual plane extending in the coronal direction. It remains asymptomatic till the periradicular pathology develops and mimics failed root canal treatment. Patient has mild signs and symptoms of pain, swelling and presence of sinus tract or a deep narrow isolated periodontal pocket along one side of the root. $\mathrm{X}$ ray may show widening or breakdown of pdl space from apical to coronal direction, localised vertical bone loss and "halo" effect of localised periradicular bone loss. Suggestive of VRF [3].

Treatment plan:Usually involves extraction. Root resection or hemi sectionmay be considered in multi rooted $\operatorname{tooth}^{[3]}$.

\section{Conclusion}

Cracks in the tooth have potential for bacterial penetration resulting in pulpal pathology. Epidemiological data reveals that the third most common cause of tooth loss is cracks or fractures in teeth. Hence, early detection, diagnosis and appropriate treatment are mandatory to prevent tooth loss.

\section{References}

[1]. Cameron CE. Cracked-tooth syndrome.J Am Dent Assoc1964;68(March):405-11.

[2]. Geurtsen W. The cracked-tooth syndrome: clinical features and case reports.IntJ Periodontol Rest Dent 1992;12:395-405

[3] . Cohen S, Blanco L, Berman L Vertical root factures; clinical and radiographic diagnosis, Journal of am dent assoc;134:434441.

[4]. Rosen H.Cracked tooth syndrome. J Prosthet Dent 1982;47(1):36-43

[5]. American association of endodontics; colleagues of excellence; "cracking the cracked tooth Code detection and treatment of variouslongitudinal tooth fractures"summer2008 available at http://www.aae.org/dentalpro/colleaguenews.htm; bonus material C.

[ 6] Christopher D Lynch, Robert J McConnell; The cracked tooth syndrome; J of Canadian DentAssoc 2002;68(8):470-5.

[7]. Khera SC, Carpenter CW, Vetter JD, Staley RN, Anatomy of cusps of posterior teeth and their fracture potential. J Prosthet Dent 1990;64:139-47

[8] American association of endodontics; colleagues of excellence; "cracking the crack tooth Code detection and treatment of various longitudinal tooth fractures" winter 1997.

[9]. Sonnomiya EK, Goncalves M, Cavalcanti MP; Masseter Muscle hypertrophy- case report;Braz Dent J;2006;17;347-350.

[10]. Sabeena Mathew, Boopathithangavel, ArjunDas ; Diagnosis of cracked tooth syndrome J of Pharm and Bio allied Sciences; 2012 Aug4(2);242-244.

[11]. Brown WS, Jacobs HR, Thompson RE, Thermal fatigue in teeth; J of Dent Res 1975;51(2);461-467.

[12]. Miller N, Penand K, Ambrosini P et al; Analysis of etiologic factors and periodontal conditions involved with 309 abfractions; $J$ ClinPeriodontol 2003; 30(9);828-832.

[13]. Mitchell H Davich;Clinical detection and decision making consideration for cracked teeth; endodontic therapyvol 4 no 2.

[14]. Alex J. Moule, Bill Kahler; Diagnosis and management of teeth with vertical root fractures;Aust Dent J;1999;44(2);75-87.

[15]. Abou-rass M Crack lines: The precursors of tooth fracture. their diagnosis and treatment..QuintesenceInt 1983;14:437-447

[16]. Wright HM Jr,Loushine RJ, Weller RN, et al. identification or resected root end dentinal cracks :a comparative study of transillumination and dyes. J Endod 2004;30:712-5

[17]. White SC, Pharoah MJ. Oral radiology, principles and interpretation (5th edn). St. Louis, MO: Mosby, $2004: \mathrm{p} 623$.

[18]. Alex J. Moule, Bill kahler; Diagnosis and management of teeth with vertical root fractures; Aust. Dent J;1999;44(2);75-87.

[19]; Sabeena Mathew, Boopathithangavel, Arjun das; Diagnosis of cracked tooth syndrome ;JOf pharm and bioallied sciences;2012;Aug 4(2);242-245.

[20] .Pitts PL, Natkin E. Diagnosis and treatment of vertical root fractures; $J$ of Endod;1983;9;338-346. 
[21]. CuljatMO,Singh RS, Brown ER,et al. ultrasound crack detection in a simulated human tooth. Dento-Maxillo-Facial Radiology 2005;34:80-5

[22]. Standler JP, Collard EW, Caputo AA; Dentinal defects caused by some twist drills and retentive pins; J Prosthet Dent 1970;24(2);185-192.

[23]. LuebkeRG.Vertical crown-root fractures in posterior tooth. Dent clin North Am 1984;28:883-894

[24]. Brannstrom M. The hydrodynamic theory of dentinal pain:sensation in preparations,caries and dentinal crack syndrome . $J$ Endod 1986;12:453-457.

[25]. Figdor D. Pain of dentinal and pulpal origin. A review for the clinician.Ann R Coll Dent Surg 1994;12:131-142

[26]. Abbott, P. Endodontics and dental traumatology. An overview modern endodontics. 1999 